\title{
Various patterns of the middle cerebral vein and preservation of venous drainage during the anterior transpetrosal approach
}

\author{
Shunsuke Shibao, MD, ${ }^{1}$ Masahiro Toda, MD, PhD, ${ }^{1}$ Maaya Orii, MD, ${ }^{1}$ Hirokazu Fujiwara, MD, PhD, ${ }^{2}$ \\ and Kazunari Yoshida, MD, PhD'
} 1Department of Neurosurgery, Keio University School of Medicine; and 2Department of Diagnostic Radiology, Keio University
Graduate School of Medicine, Tokyo, Japan

\begin{abstract}
OBJECTIVE The drainage of the superficial middle cerebral vein (SMCV) has previously been classified into 4 subtypes. Extradural procedures and dural incisions during the anterior transpetrosal approach (ATPA) may interrupt the route of drainage from the SMCV. In this study, the authors examined the relationship between anatomical variations in the SMCV and the corresponding surgical modifications to the ATPA that are necessary for venous preservation.
\end{abstract}

METHODS This study included 48 patients treated via the ATPA in whom the SMCV was examined using 3D CT venography. The drainage patterns of the SMCV were classified into 3 types: cavernous or absent (Type 1), sphenobasal (Type 2), and sphenopetrosal (Type 3). Type 2 was subdivided into medial (Type 2a) and lateral (Type 2b), and Type 3 was subdivided into vein (Type 3a), vein and sinus (Type 3b), and sinus (Type 3c). The authors performed 3 ATPA modifications to preserve the SMCV: epidural anterior petrosectomy with subdural visualization of the sphenobasal vein (SBV), modification of the dural incision, and subdural anterior petrosectomy. Standard ATPA can be performed with Type 1, Type 2a, and Type $3 a$ drainage. With Type $2 b$ drainage, an epidural anterior petrosectomy with subdural SBV visualization is appropriate. The dural incision should be modified in Type 3b. With Type 3c, a subdural anterior petrosectomy is required.

RESULTS The frequency of each type was $68.7 \%$ (33/48) in Type 1, 8.3\% (4/48) in Type 2a, 4.2\% (2/48) in Type 2b, $14.6 \%(7 / 48)$ in Type 3a, 2.1\% (1/48) in Type 3b, and 2.1\% (1/48) in Type 3c. No venous complications were found.

CONCLUSIONS The authors propose an SMCV modified classification based on ATPA modifications required for venous preservation.

http://thejns.org/doi/abs/10.3171/2015.1.JNS141854

KEY WORDS anterior transpetrosal approach; superficial middle cerebral vein; Hacker's classification; venous preservation; anatomy

$\mathrm{T}$ HE superficial middle cerebral vein (SMCV) usually runs inferiorly and anteriorly along the sylvian fissure and leads into the sphenoparietal sinus or directly into the cavernous sinus (CS). ${ }^{3,5,13,25,27}$ Hacker classified the SMCV drainage pattern into 4 types: 1) sphenoparietal sinus, 2) sphenobasal vein (SBV), 3) sphenopetrosal vein, and 4) cortical veins with the absence of a definite SMCV. ${ }^{9}$

The anterior transpetrosal approach (ATPA) is best suited for upper petroclival lesions located anterior and superior to the internal auditory canal and superior to the inferior petrosal sinus. ${ }^{15,16}$ Epidural procedures and dural incision of the middle temporal fossa in ATPA may interrupt drainage routes from the SMCV.

It has been demonstrated that surgical interruption of the SMCV leads to temporal lobe damage., ${ }^{1,17} \mathrm{We}$ need to consider postoperative changes in the pattern of SMCV drainage and the possibility of complications due to surgical interruption of venous drainage at the temporal skull base. To investigate the relationship between anatomical variations in the SMCV and modification to the ATPA, we have demonstrated the modifications made to the ATPA

ABBREVIATIONS ATPA = anterior transpetrosal approach; CS = cavernous sinus; CTV = CT venography; DSA = digital subtraction angiography; GPN = greater petrosal nerve; SBV = sphenobasal vein; SMCV = superficial middle cerebral vein; SPS = superior petrosal sinus.

ACCOMPANYING EDITORIAL See pp 429-431. DOI: 10.3171/2015.3.JNS15141.

SUBMITTED August 11, 2014. ACCEPTED January 20, 2015.

INCLUDE WHEN CITING Published online August 28, 2015; DOI: 10.3171/2015.1.JNS141854. 
during our experience with patients who had petroclival lesions, and we have reorganized Hacker's classification to ensure venous preservation.

\section{Methods}

Our institutional review board approved this study, and written informed consent for imaging examinations was obtained from all patients.

\section{Study Population}

This study consisted of 48 patients treated via the ATPA in our hospital between 2010 and 2014. These included 20 male and 28 female patients, $12-75$ years (mean age 52.8 years). We modified the ATPA in patients in whom the SMCV could have been injured by the procedure. The drainage patterns of the SMCV were examined on the operative side in these patients by using 3D CT venography (CTV).

\section{The 3D CTV Protocol}

All examinations were performed with a CT scanner (Aquilion one; Toshiba Medical Systems) equipped with 320 detector rows (each $0.5 \mathrm{~mm}$ wide) covering $16 \mathrm{~cm}$ of volume per rotation. Initially, a test bolus scan was performed at the level of the carotid bulb to determine the optimal timing of dynamic scans using an intravenous injection of nonionic contrast medium $(20 \mathrm{ml})$ at a rate of $5 \mathrm{ml} / \mathrm{second}$, followed by saline $(20 \mathrm{ml})$. Subsequently, CT digital subtraction angiography (DSA) scans were obtained after a 50-ml bolus injection of contrast medium at the rate of $5 \mathrm{ml} / \mathrm{second}$, followed by saline $(20 \mathrm{ml})$. The CT DSA scans consisted of a volume scan before the arrival of contrast media to provide an unenhanced mask volume data set for subsequent bone subtraction, continuous volume scans over 10 seconds (rotation time 1 second), and 4 intermittent scans with an interval of 4 seconds as venous phases. Other scan parameters were as follows: field of view $25 \mathrm{~cm}$; slice thickness $0.5 \mathrm{~mm}$; tube voltage $80 \mathrm{kV}$; and current-time product $350 \mathrm{~mA}$ (first and last phases) and $100 \mathrm{~mA}$ (other phases). Multiphase and multidirectional CT DSA images were generated for venous evaluation by using maximum intensity projection and volume-rendering methods. The volume-rendering images of arterial and venous phases were superimposed on bone images of the skull base to improve the recognition of skull base vascular anatomy. The 3D CTV scans were evaluated by the same neurosurgeon on 2 separate occasions.

\section{Classification of SMCV}

Variations among the SMCVs in 48 sides in the ATPA cases were classified into 3 types and 6 subtypes based on preoperative 3D CTV and intraoperative findings. Hacker classified the SMCV into 4 groups; CS, sphenobasal, sphenopetrosal, and absent type. ${ }^{9}$ We rearranged Hacker's classification for the purpose of venous preservation in the ATPA (Figs. 1-3). Cavernous and absent types were grouped together (Type 1) (Fig. 1); sphenobasal type was divided into medial and lateral types (Types $2 \mathrm{a}$ and $2 \mathrm{~b}$ ) (Fig. 2); and sphenopetrosal type was divided into vein, vein and sinus, and sinus types (Types 3a, 3b, and 3c) (Fig. 3). "Sinus" means the vein going through the interdural space. In the case of Type 1, the vein enters the sphenoparietal sinus to drain directly into the CS (Fig. 1A and C) or the vein may be absent (Fig. 1B and D). In Type 2 , the venous drainage runs parallel to the lesser wing of the sphenoid and drains into the pterygoid venous plexus through the medial (Fig. 2A and C) or lateral (Fig. 2B and D) side of the foramen ovale. In Type 3, the vein passes posterior along the floor of the middle cranial fossa to drain into the transverse sinus or the posterior part of the superior petrosal sinus (SPS) (Fig. 3A-D). In this type, we intraoperatively identified whether the vein was the sphenopetrosal vein or sinus and confirmed the subtype by visualization of the SMCV from the subdural side. Additionally, we encountered the vein undefined by Hacker's classification (Fig. 3E).

\section{Modification of ATPA for Venous Preservation}

In the case of Type 1 , Type $2 \mathrm{a}$, and Type $3 \mathrm{a}$, we performed a standard epidural ATPA (Fig. 4A-D, F). As for other types of SMCV, we implemented 3 types of modification to the ATPA for venous preservation: epidural anterior petrosectomy with subdural visualization of the SBV for lateral type SBV (Type 2b) (Fig. 4E); dural incision modification for the sphenopetrosal vein draining into the
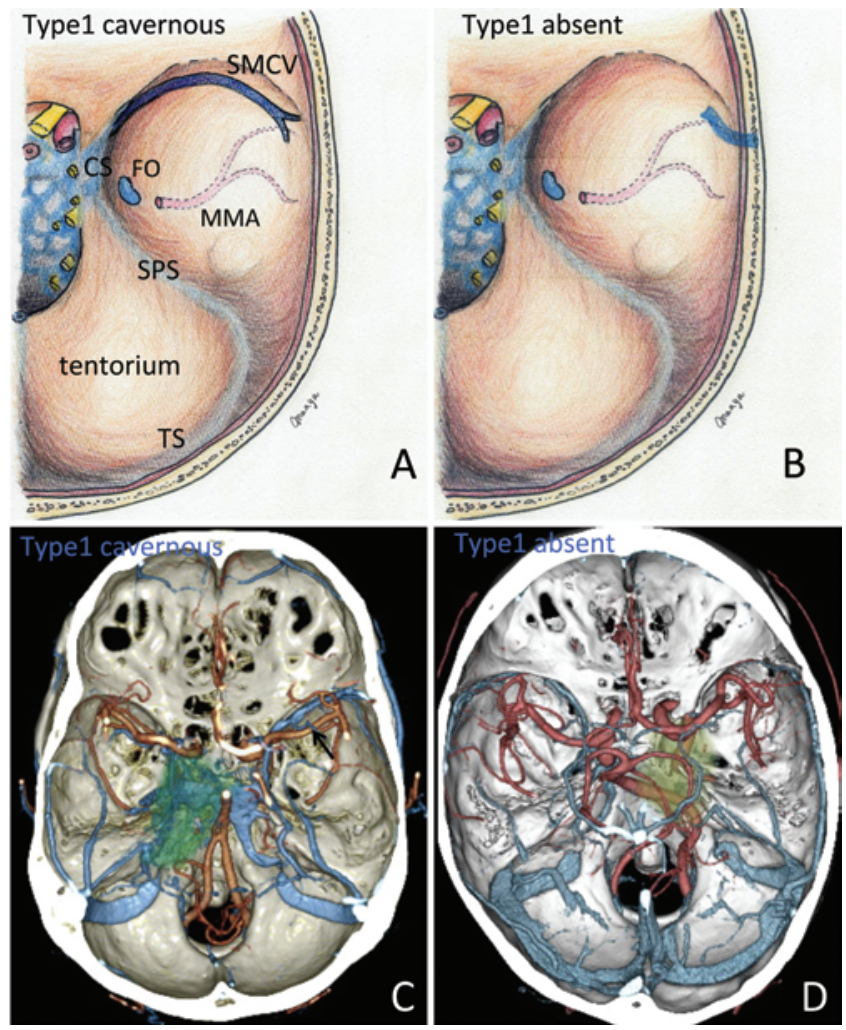

FIG. 1. Type 1 (cavernous or absent type) includes CS (A) or absent type (B) in Hacker's classification. C and D: Representative 3D CTV scans showing the cavernous type (C) and absent type (D) SMCV. Arrow in $\mathrm{C}$ designates the vein showing classification. $\mathrm{FO}=$ foramen ovale; MMA = middle meningeal artery; TS = transverse sinus. Drawings in A and $B$ copyright Maaya Orii. Published with permission. Figure is available in color online only. 
sphenopetrosal sinus in the middle fossa (Type 3b) (Fig. $4 \mathrm{G}$ ); and subdural anterior petrosectomy for the sphenopetrosal sinus in the middle fossa (Type 3c) (Fig. 4H). We did not encounter veins in which the transition from the sphenopetrosal vein to the sinus is found (as in Type 3b), where the dura mater could not be peeled from the middle fossa (Fig. 4I).

\section{Procedure of Each Modification}

Epidural Anterior Petrosectomy With Subdural Visualization of the SBV

After the SBV was subdurally visualized by retracting the anterior part of the temporal lobe, the temporal basal dura mater posterior to the SBV was cut and the dura mater medial to the greater petrosal nerve (GPN) and along the edge of the petrous apex was dissected to expose the petrous apex epidurally (Fig. 4E).

\section{Modification of the Dural Incision}

We first confirmed the part at which the vein ran from the sphenopetrosal vein into the temporal basal dura as a sinus from the subdural side. We then cut the basal dura of the temporal lobe anterior to that region and medial to the sinus (Fig. 4G).

\section{Subdural Anterior Petrosectomy}

After we confirmed that the drainage route of the SMCV was a sinus from the subdural side, we identified the petrous apex from the subdural side, judging by the surrounding structures and using navigation and/or GPN monitoring. The dura was then cut medial to the GPN and along the edge of the petrous apex, and the petrous apex was drilled out (Fig. 4H).

\section{Results}

Table 1 summarizes the distribution of each drainage type and shows the comparison with Hacker's classification among 48 patients treated using the ATPA. The frequency of each type was as follows: Type 1, 68.7\% (33/48); Type 2a, $8.3 \%$ (4/48); Type 2b, $4.2 \%$ (2/48); Type 3a, $14.6 \%$ (7/48); Type 3b, $2.1 \%$ (1/48), and Type 3c, $2.1 \%$ (1/48). No venous complications were found in these cases. We have summarized the relationship between variations in the SMCV and modifications to the ATPA required for venous preservation in Table 2.

\section{Illustrative Cases}

We have illustrated each ATPA modification used for venous preservation with the following representative cases.

\section{Case 1: Epidural Anterior Petrosectomy With Subdural Visualization of SBV (Type $2 b$ )}

A 34-year-old woman presented with headache. Imaging studies revealed a lesion in the prepontine cistern (Fig. 5A). Preoperative 3D CTV revealed that the SMCV drained into the posterolateral side of the foramen ovale (Fig. 5B). To preserve the drainage route, the SBV was visualized from the subdural side and the temporal basal dura mater was cut posterior to the SBV. The middle men-
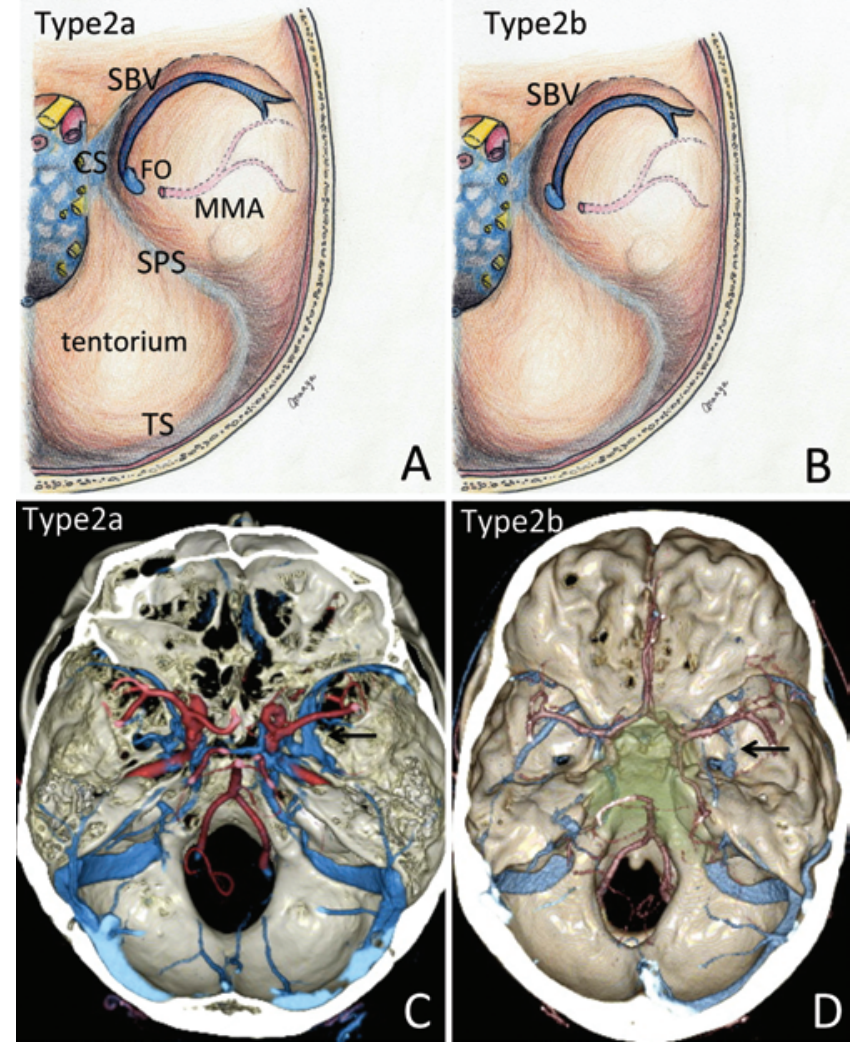

FIG. 2. Type 2 (sphenobasal type) includes the SBV type in Hacker's classification. A: Type 2 a has drainage to the medial side of the foramen ovale. B: Type $2 b$ has drainage to the posterolateral side of the ovale. C and D: Representative 3D CTV scans showing Type 2a (medial) (C) and Type $2 b$ (lateral) (D). Arrows designate the vein showing classification. Drawings in A and B copyright Maaya Orii. Published with permission. Figure is available in color online only.

ingeal artery was preserved to avoid exposing the foramen ovale, and the posterior part of the temporal lobe was retracted with the dura mater. The dura mater was incised medial to the GPN and along the edge of the petrous apex, exposing the petrous apex (Fig. 5C-E). The tumor was completely removed, and no venous complications were found postoperatively (Fig. 5F).

\section{Case 2: Modification of Dural Incision (Type 3b)}

A 31-year-old man presented with left facial hypesthesia. MRI revealed a left parasellar chordoma (Fig. 6A). Preoperative angiography showed that the venous drainage of the SMCV ran through the middle fossa into the sphenopetrosal vein or sinus (Fig. 6B). The patient initially underwent surgery using a subtemporal epi- and interdural approach, and the tumor was subtotally excised. A second operation in which a subtemporal subdural approach was used was performed 1 month later. From the subdural side, we first confirmed that the vein ran from the sphenopetrosal vein into the temporal basal dura as a sinus (Fig. 6C). After we identified the transitional region from the vein to the sinus subdurally, we cut the temporal lobe basal dura anterior to that region and medial to the sinus (Fig. $6 \mathrm{D}$ and $\mathrm{E}$ ). The soft compartment of the tumor was totally removed. Postoperatively, no venous complications were observed (Fig. 6F). 

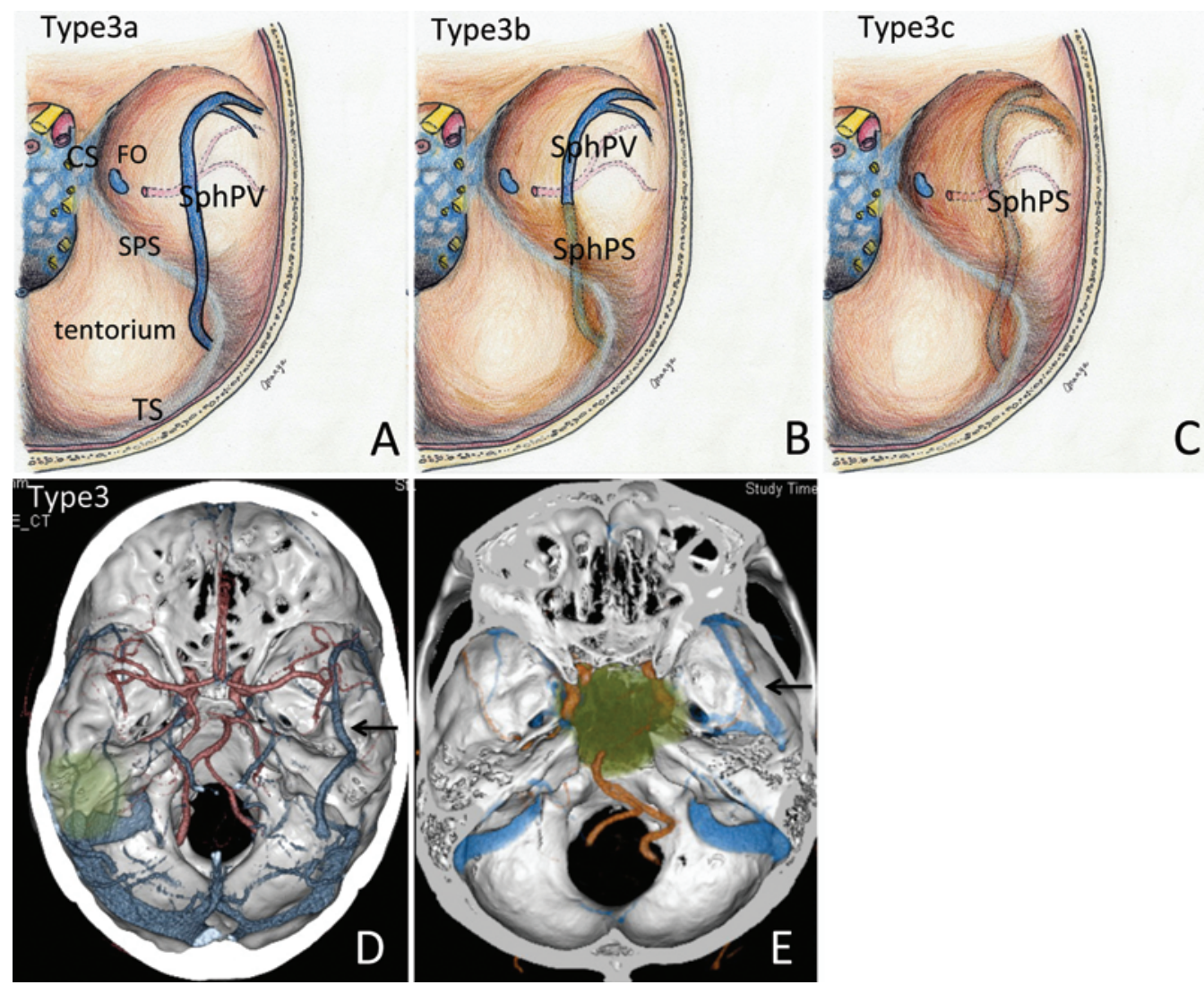

FIG. 3. Type 3 (middle fossa type) includes the sphenopetrosal type by Hacker's classification (A-D) and the sinus that passes posteriorly along the middle cranial fossa and cannot be included in Hacker's classification due to its unique course (E). In this type, we should first confirm the location of the vein or sinus from the subdural side. A: Type 3a is the vein type. B: Type 3b is the vein and sinus type, in which the basal dura mater can be peeled back from the middle fossa, and the dural incision should be modified according to the transitional region from vein to sinus. C: Type $3 \mathrm{c}$ is the sinus type, in which the basal dura cannot be peeled back from the middle fossa. D and E: Representative 3D CTV scans showing Type 3 (petrosal type) (D) and the other sinus undefined by Hacker's classification (E). Arrows designate the vein included in Type 3 in our classification; solid blue line denotes the venous part of the SMCV; and transparent blue line denotes the sinus part of the SMCV. SphPS = sphenopetrosal sinus; SphPV = sphenopetrosal vein. Drawings in A-C copyright Maaya Orii. Published with permission. Figure is available in color online only.

\section{Case 3: Subdural Anterior Petrosectomy (Type 3c)}

A 26-year-old man presented with headache and diplopia. The patient had undergone subtotal excision of a clival giant cell tumor 8 months prior via an endoscopic endonasal approach and had received stereotactic radiosurgery to treat the residual tumor. Imaging studies revealed a solid lesion, located in the clivus, pressing on the brainstem posteriorly (Fig. 7A). Preoperative 3D CTV showed that the SMCV ran through the middle fossa posterolaterally and turned anteromedially to the foramen ovale (Fig. 7B). The patient underwent an ATPA. We confirmed that the drainage route of the SMCV was a sinus subdurally. Although we tried to peel back the temporal basal dura from the skull base, it was impossible to expose the petrous apex epidurally because of the emissary vein from the dural sinus of the middle fossa. We identified the petrous apex from the subdural side, judging by the surrounding structures and using navigation (Fig. 7C). The dura was then cut medial to the GPN and along the edge of the petrous apex, and the petrous apex was drilled out (Fig. 7D and E). The subdural tumor was partially removed. Postoperatively the patient developed mild worsening left hemiparesis, but no venous complications (Fig. 7F).

\section{Discussion}

In 1974, Hacker classified the SMCV into 4 subtypes by angiography: CS, sphenobasal, sphenopetrosal, and absent type. ${ }^{9}$ In 2000, Suzuki and Matsumoto reported another classification of the SMCV based on embryological development by using 3D CTV. ${ }^{25}$ Although these classifications were considered important for preoperative evaluation, a detailed explanation of the appropriate surgical procedure for each of the venous variations has not yet been reported. In this study we report a new classification of the SMCV for surgical procedures, especially ATPA.

\section{Basic Concept of the ATPA}

The ATPA was first described in 1985 for upper petroclival lesions located anterior and superior to the internal auditory canal and superior to the inferior petrosal sinus. ${ }^{15,16}$ The standard ATPA for skull base lesions fundamentally requires epidural subtemporal procedures to expose the petrous apex adequately (Fig. 4A).,10 The 

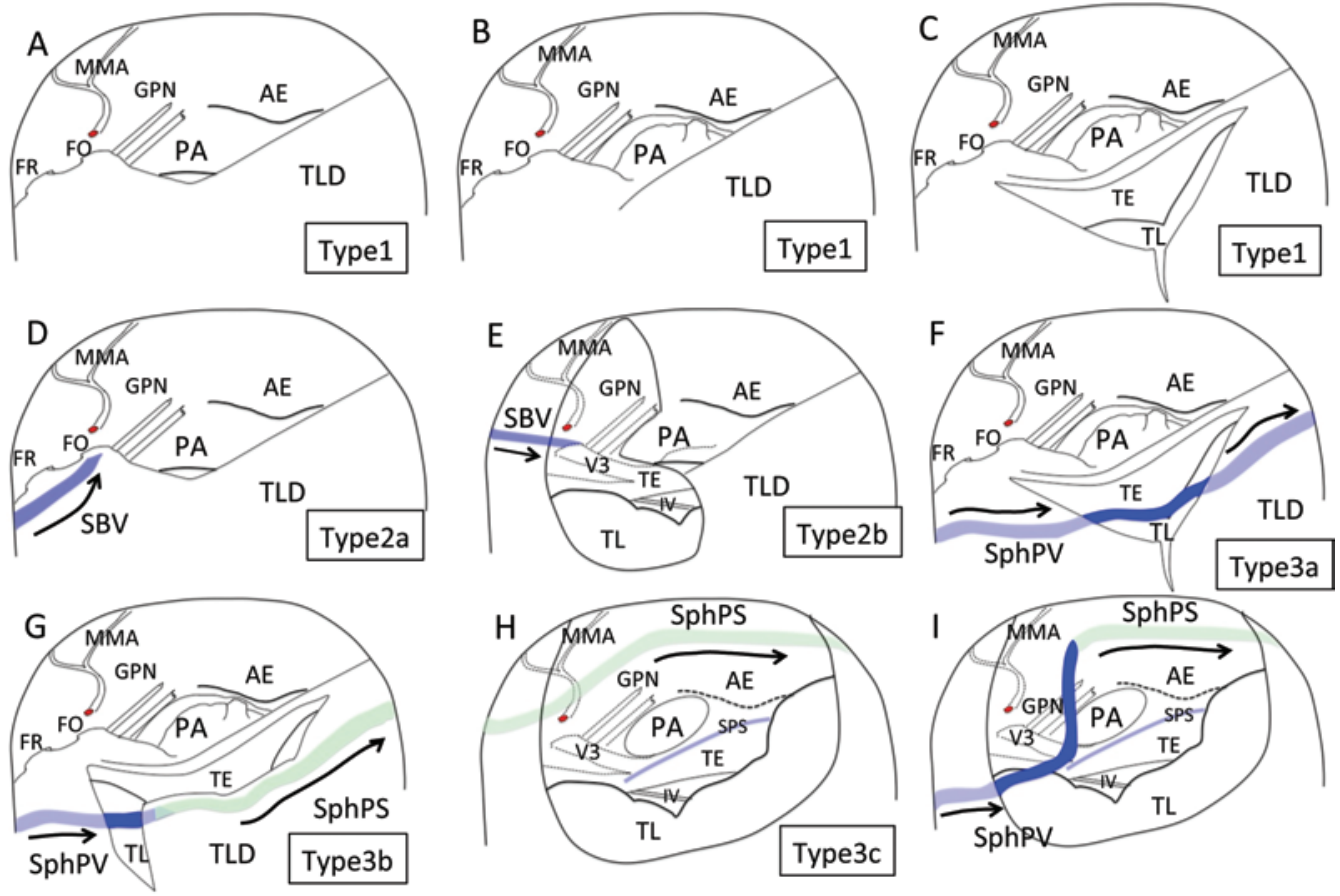

FIG. 4. Schema of venous preservation for each type. A-C: Standard steps in the ATPA. A: Exposure of the petrous apex (PA). B: Drilling of the PA. C: Dural incision to ligate the SPS. D and E: Comparison of Type $2 a$ and Type $2 b$. In Type $2 a$, we can expose the PA because the SBV is medial to the foramen ovale (D). In Type $2 b$, we should perform an epidural anterior petrosectomy with subdural visualization of the SBV (E). F-H: Comparison of Types 3a-c. In Type 3a, we can incise the temporal basal dura in the standard way, as in Type $1(F)$. In Type 3b, we should modify the dural incision to avoid injury of the sphenopetrosal sinus (modification of dural incision) (G). In Type 3c, the temporal basal dura cannot be peeled back, and the subdural exposure and drilling of the PA are required (subdural anterior petrosectomy) $(\mathrm{H})$. I: A case in which the transition from the sphenopetrosal vein to the sinus is like Type $3 \mathrm{~b}$ (panel G), but the temporal lobe dura cannot be peeled off. In the subdural view, the sphenopetrosal vein is bridged to the dural sinus. The cranial nerves are numbered. Arrows denote the direction of venous flow. AE = arcuate eminence; $\mathrm{FR}=$ foramen rotundum; $\mathrm{TE}=$ tentorium; $\mathrm{TL}$ = temporal lobe; $\mathrm{TLD}=$ temporal lobe dura; $\mathrm{V} 3$ = mandibular nerve. Figure is available in color online only.

middle meningeal artery is coagulated and cut. The GPN is dissected in the interdural layer to preserve the nerve. ${ }^{12}$ To achieve improved exposure of the petroclival region through the middle fossa corridor, and to gain a wider operative field, the petrous apex must be totally resected (Fig. 4B). The dura of the temporal lobe and posterior fossa is then cut to ligate the SPS and the tentorium (Fig. 4C). Various modifications of the ATPA to improve exposure of the petroclival region have been described, including additional zygomatic osteotomy, anterior mobilization of

TABLE 1. Distribution of SMCV variants among 48 patients treated using the ATPA: comparison between our modified classification and Hacker's classification

\begin{tabular}{|c|c|c|c|}
\hline Modified Classification & $\begin{array}{c}\text { Corresponding } \\
\text { Hacker's } \\
\text { Classification }\end{array}$ & $\begin{array}{c}\text { No. of } \\
\text { Patients }\end{array}$ & $\%$ \\
\hline Type 1 (cavernous or absent) & CS or absent & 33 & 68.7 \\
\hline Type 2a (sphenobasal: medial) & Sphenobasal & 4 & 8.3 \\
\hline Type 2b (sphenobasal: lateral) & & 2 & 4.2 \\
\hline Type 3a (sphenopetrosal: vein) & Sphenopetrosal & 7 & 14.6 \\
\hline $\begin{array}{l}\text { Type 3b (sphenopetrosal: vein \& } \\
\text { sinus) }\end{array}$ & & 1 & 2.1 \\
\hline Type 3c (sphenopetrosal: sinus) & & 1 & 2.1 \\
\hline
\end{tabular}

the mandibular nerve, and an extensive middle fossa approach. ${ }^{2,6,7,29}$

\section{Veins and the ATPA}

There have been several reports regarding veins and their impact on the ATPA. Guppy et al. reported a relationship between the venous drainage system of the inferior and lateral temporal lobes and the choice of lateral cranial base approach. ${ }^{8}$ They stressed the importance of the vein of Labbé and venous lake of the tentorium. Sakata et al. focused on temporal bridging veins other than the vein of Labbé and emphasized the importance of reducing any likelihood of venous complications during surgery. ${ }^{21}$ Hayashi et al. reported a relationship between the venous pattern encountered in the ATPA and surgical results. ${ }^{10}$ They concluded that venous drainage from the CS should

TABLE 2. Modification of the ATPA according to SMCV variation

\begin{tabular}{ll}
\hline \multicolumn{1}{c}{ Modification of the ATPA } & Variation of SMCV \\
\hline $\begin{array}{l}\text { 1. Standard ATPA } \\
\begin{array}{l}\text { 2. Epidural anterior petrosectomy w/ subdural } \\
\text { visualization of SBV }\end{array}\end{array}$ & Types 1, 2a, \& 3a $2 b$ \\
\hline 3. ATPA w/ modification of dural incision & Type 3b \\
\hline 4. Subdural anterior petrosectomy & Type 3c \\
\hline
\end{tabular}



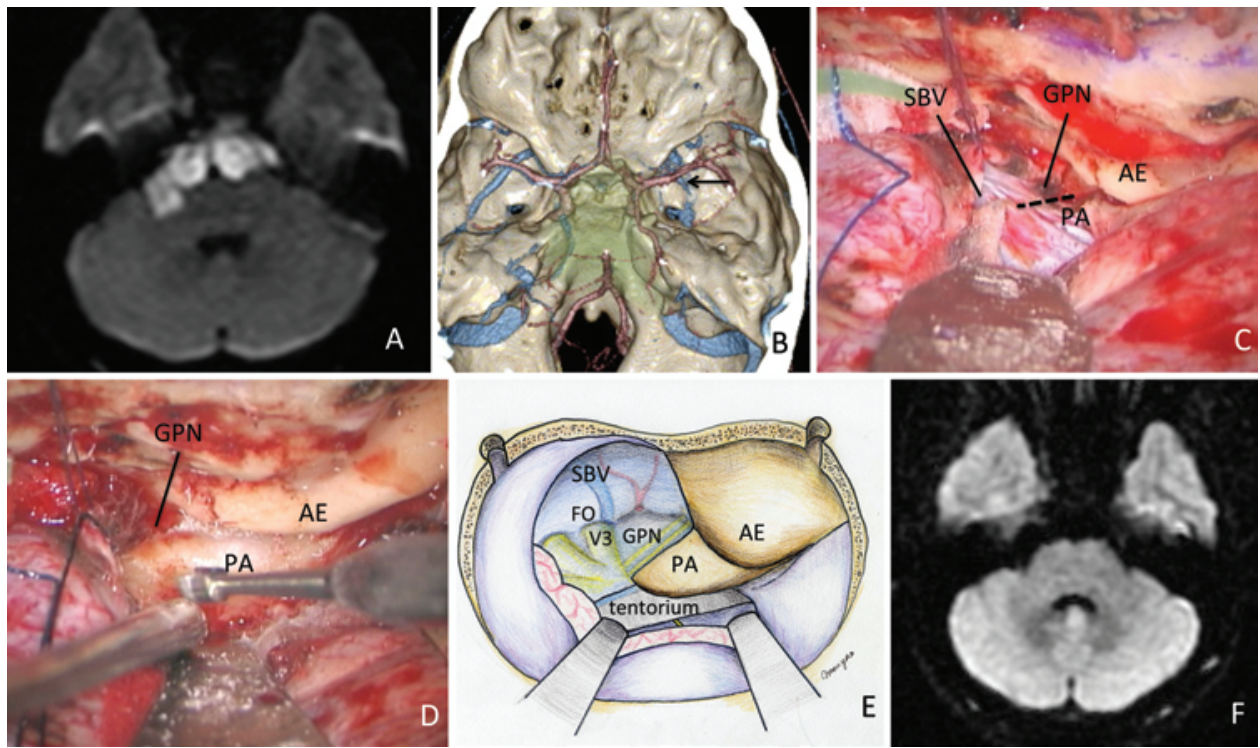

FIG. 5. Illustrative Case 1. A case of epidural anterior petrosectomy with subdural visualization of the SBV. A: Preoperative diffusion-weighted MR image showing an epidermoid cyst in the prepontine cistern and the right cerebellopontine angle. B: Preoperative 3D CTV scan showing that the SMCV drained into the posterolateral side of the foramen ovale (arrow). C and D: Intraoperative photographs showing that the dura mater medial to the GPN was cut $(C)$ and the petrous apex was drilled while preserving the SBV (D). E: Schema of epidural anterior petrosectomy with subdural visualization of the SBV. F: Diffusion-weighted MRI study obtained after definitive surgery showing total removal and no venous complications. Drawing in E copyright Maaya Orii. Published with permission. Figure is available in color online only.

be preserved into the pterygoid venous plexus, rather than into the inferior petrous sinus.

Venous congestion leads to brain injury. ${ }^{17}$ Some authors have reported postoperative venous infarction at a rate of $2.6 \%-13 \% .^{1,14,20}$ Cerebral venous circulation disturbances can cause unexpected severe postoperative complications in neurosurgical practice. ${ }^{19}$ We performed 3D CTV to evaluate venous draining patterns preoperatively, which is very useful in considering preoperative strategies to preserve the SMCV. The usefulness of this procedure was stressed in a previous report. ${ }^{10}$

\section{Classification of the SMCV}

We have reorganized Hacker's classification based on
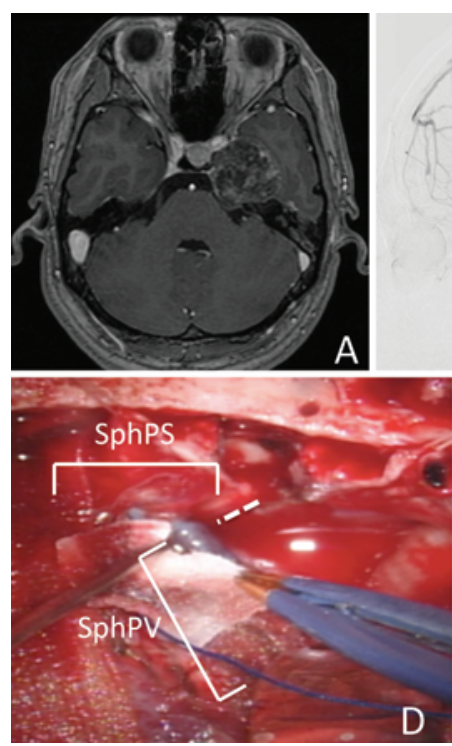

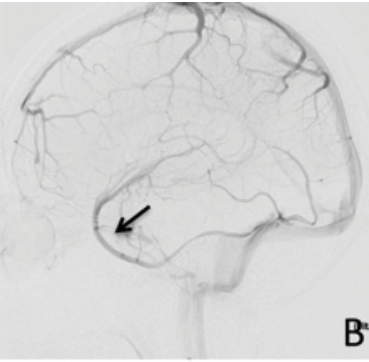

B
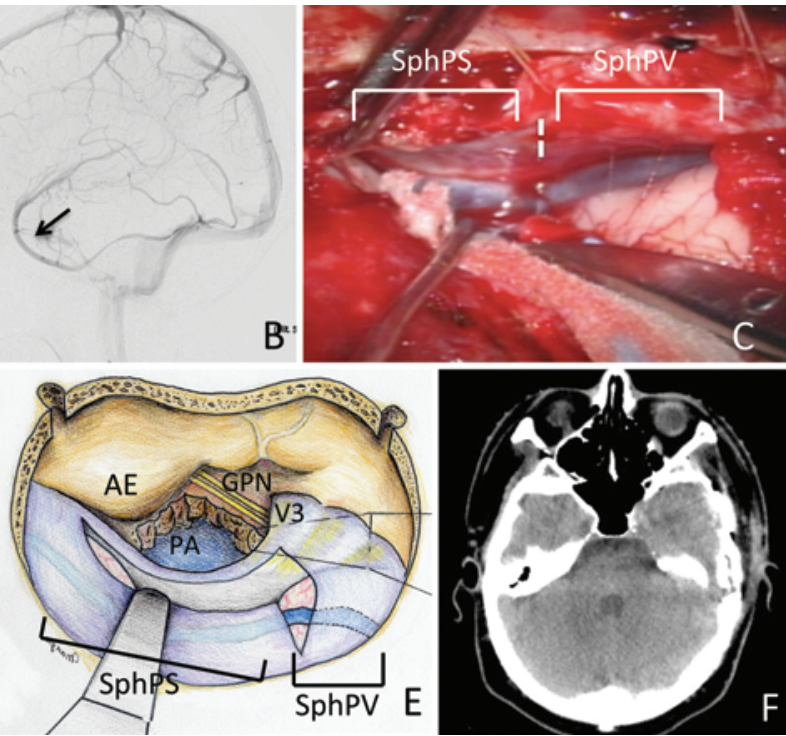

FIG. 6. Illustrative Case 2. A case requiring modification of the dural incision. A: Preoperative contrast-enhanced T1-weighted MR image showing a left parasellar chordoma. B: Preoperative venogram showing the SMCV draining through the sphenoparietal vein or sinus (arrow). C and D: Intraoperative photographs showing the transitional region from the vein to the sinus. The dotted line shows the incision of the temporal lobe dura anterior to the transitional region and medial to the sinus. E: Schema of dural incision modification. F: Contrast-enhanced CT scan obtained after definitive surgery showing total removal and no venous complications. Drawing in E copyright Maaya Orii. Published with permission. Figure is available in color online only. 

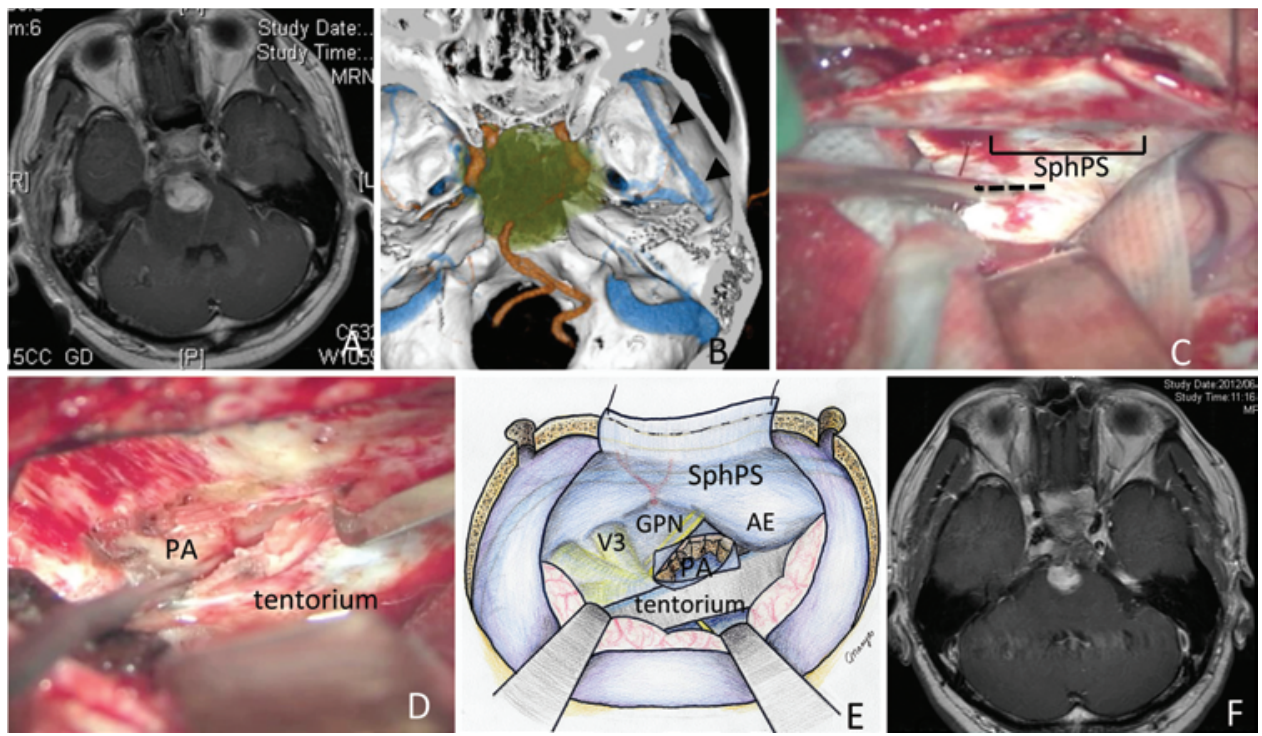

FIG. 7. Illustrative Case 3. A case requiring subdural anterior petrosectomy. A: Preoperative contrast-enhanced T1-weighted MR image showing a clival tumor indenting the brainstem posteriorly. B: Preoperative 3D CTV showing that the SMCV ran posterolaterally and turned anteromedially to the foramen ovale (arrowheads). C and D: Intraoperative photographs showing the site of dural incision (dotted line) (C) and the petrous apex visualized from the subdural side (D). E: Schema of subdural anterior petrosectomy. F: Contrast-enhanced T1-weighted MR image obtained after definitive surgery showing subtotal excision and no venous complications. Drawing in E copyright Maaya Orii. Published with permission. Figure is available in color online only.

our own experiences (Fig. 1-3). Type 1 corresponds to the CS or absent type (Fig. 1), Type 2 to the sphenobasal type (Fig. 2), and Type 3 to the sphenopetrosal type (Fig. 3) in Hacker's classification. Type 2 is classified into 2 subtypes: medial (Type 2a) and lateral (Type 2b), depending on the side of the foramen ovale through which the vein drains (Fig. 2). Type 3 is classified into 3 subtypes: vein (Type 3a), vein and sinus (Type 3b), and sinus (Type 3c) (Fig. 3). Especially in Type $2 b$, Type $3 b$, and Type $3 c$, we should consider modification of the ATPA for venous preservation (Fig. 4A-H): an epidural anterior petrosectomy with subdural visualization of the SBV for Type $2 \mathrm{~b}$ (Fig. 4E); modification of the middle fossa dura for Type $3 \mathrm{~b}$ (Fig. 4F); and subdural anterior petrosectomy for Type 3c (Fig. 4H).

In Type 1, a standard ATPA is appropriate (Fig. 4A-C). If the SMCV is absent, however, the vein of Labbe and temporal bridging veins tend to develop, and these veins need to be treated carefully. ${ }^{28}$

Preservation of the SBV in ATPA has been described by Ichimura et al. ${ }^{11}$ They explained the procedure for epidural anterior petrosectomy with subdural visualization of the SBV (Fig. 4E). Briefly, after the SBV was visualized subdurally by retracting the anterior part of the temporal lobe, the temporal basal dura mater posterior to the SBV was cut and the dura mater medial to the GPN and along the edge of the petrous apex was dissected to expose the petrous apex epidurally.

However, we have encountered cases in which a standard ATPA could be performed because the SBV drained into the medial side of the foramen ovale and did not interrupt the corridor (Type 2a) (Fig. 4D). We have distinguished cases in which the SBV drains into the medial side of the foramen ovale (Type 2a) from those draining into the lateral side (Type $2 b$ ).

In Type 3, it is important to examine from the subdu- ral side whether the SMCV is a vein, both a vein and a sinus, or a sinus, because the difference between a vein or a sinus cannot be determined preoperatively. In the case of both vein and sinus, the dural incision should be modified to avoid injuring the sinus part in the sphenopetrosal type (Type 3b) (Fig. 4G). In the case of a sinus (Type 3c), a subdural anterior petrosectomy should be considered (Fig. 4H). The subdural anterior petrosectomy has been reported by Steiger et al. ${ }^{24}$ They reported a subdural anterior petrosectomy as follows: after opening of the basal dura mater, the tentorium is incised behind the entrance of the trochlear nerve toward the SPS, and the dura is stripped from the petrous pyramid. Drilling then starts at the petrous ridge and proceeds laterally and ventrally. Our concept for the subdural anterior petrosectomy is similar to the approach reported by them. A navigation system and monitoring by GPN stimulation are useful for subdural identification of the petrous apex. ${ }^{26}$

\section{Modification of the ATPA}

We performed 3 modifications to the ATPA: the epidural anterior petrosectomy with subdural visualization of the SBV (Figs. 4E and 5E); the ATPA with modification of the dural incision (Figs. 4G and 6E); and the subdural anterior petrosectomy (Fig.s $4 \mathrm{H}$ and 7E). Table 2 summarizes the relationship between the modification of the ATPA and each type of SMCV. We have not yet experienced a case with the transition from the sphenopetrosal vein to the sinus as in Type 3b, where the dura cannot be peeled from the middle fossa (Fig. 4I). In such a case, although we could consider a subdural anterior petrosectomy, the vein could tether to the dural sinus as a bridging vein and interrupt the operative corridor. In this case, a staged "intentional" bridging vein ligation or retrosigmoid approach could be considered ${ }^{18,22,23}$ Staged intentional bridging vein 
ligation is based on the idea that the cutting of the bridging veins from the temporal lobe to the floor of the middle cranial fossa in the first stage can lead to redirection of the venous drainage over time. However, we should still consider the risk of venous infarction in this procedure.

\section{Conclusions}

We have described the surgical modification of the ATPA for vein preservation: epidural anterior petrosectomy with subdural visualization of the SBV, ATPA with dural incision modification, and the subdural anterior petrosectomy. We have also proposed a new SMCV classification based on our experience: cavernous or absent (Type 1), sphenobasal (Type 2), and middle fossa type (Type 3) to ensure venous preservation.

\section{References}

1. Almefty O, Krist AF: The dangerous veins, in Hakuba A (ed): Surgery of the Intracranial Venous System. Berlin: Springer, 1996, pp 338-345

2. Deda H, Ugur HC: Zygomatic anterior subtemporal approach for lesions in the interpeduncular cistern. Skull Base 11:257264, 2001

3. Di Chiro G: Angiographic patterns of cerebral convexity veins and superficial dural sinuses. Am J Roentgenol Radium Ther Nucl Med 87:308-321, 1962

4. Dolenc VV: Frontotemporal epidural approach to trigeminal neurinomas. Acta Neurochir (Wien) 130:55-65, 1994

5. Dolenc VV: Petroclival meningiomas, in Microsurgical Anatomy and Surgery of the Skull Base. New York: Springer, 2003

6. Fujitsu K, Kuwabara T: Zygomatic approach for lesions in the interpeduncular cistern. J Neurosurg 62:340-343, 1985

7. Fukushima T, Day JD, Hirahara K: Extradural total petrous apex resection with trigeminal translocation for improved exposure of the posterior cavernous sinus and petroclival region. Skull Base Surg 6:95-103, 1996

8. Guppy KH, Origitano TC, Reichman OH, Segal S: Venous drainage of the inferolateral temporal lobe in relationship to transtemporal/transtentorial approaches to the cranial base. Neurosurgery 41:615-620, 1997

9. Hacker H: Normal supratentorial veins and dural sinus, in Newton TH, Potts DG (eds): Radiology of the Skull and Brain. Saint Louis: Mosby, 1974

10. Hayashi N, Sato H, Tsuboi Y, Nagai S, Kuwayama N, Endo $\mathrm{S}$ : Consequences of preoperative evaluation of patterns of drainage of the cavernous sinus in patients treated using the anterior transpetrosal approach. Neurol Med Chir (Tokyo) 50:373-377, 2010

11. Ichimura S, Yoshida K, Kagami H, Inaba M, Orii M, Kitamura Y, et al: Epidural anterior petrosectomy with subdural visualization of sphenobasal vein via the anterior transpetrosal approach-technical case report. Neurosurg Rev 35:609-614, 2012

12. Ichimura S, Yoshida K, Sutiono AB, Horiguchi T, Sasaki H, Kawase T: Greater petrosal nerve schwannomas-analysis of four cases and review of the literature. Neurosurg Rev 33:477-482, 2010

13. Jones F: Buchanan's Manual of Anatomy. London: Bailliere, Tindall \& Cox, 1950

14. Kageyama Y, Watanabe K, Kobayashi S, Nakamura H, Satoh A, Watanabe Y, et al: Postoperative brain damage due to cerebral vein disorders resulting from the pterional approach, in Hakuba (ed): Surgery of the Intracranial Venous System. Berlin: Springer, 1996, pp 311-315

15. Kawase T, Shiobara R, Toya S: Anterior transpetrosaltranstentorial approach for sphenopetroclival meningiomas: surgical method and results in 10 patients. Neurosurgery 28:869-876, 1991
16. Kawase T, Toya S, Shiobara R, Mine T: Transpetrosal approach for aneurysms of the lower basilar artery. J Neurosurg 63:857-861, 1985

17. Nakase H, Shin Y, Nakagawa I, Kimura R, Sakaki T: Clinical features of postoperative cerebral venous infarction. Acta Neurochir (Wien) 147:621-626, 2005

18. Nanda A, Ambekar S: Retrosigmoid approach for resection of petroclival meningioma. Neurosurg Focus 36 (1 Suppl):1, 2014

19. Otsuka H, Nakase H, Nagata K, Ueda K, Kempski O, Sakaki T: Effect of age on cerebral venous circulation disturbances in the rat. J Neurosurg 93:298-304, 2000

20. Saito F, Haraoka J, Ito H, Nishioka H, Inaba I, Yamada Y: [Venous complications in pterional approach; About frontotemporal bridging veins.] Surg Cereb Stroke 26:237-241, 1998 (Jpn)

21. Sakata K, Al-Mefty O, Yamamoto I: Venous consideration in petrosal approach: microsurgical anatomy of the temporal bridging vein. Neurosurgery 47:153-161, 2000

22. Samii M, Tatagiba M, Carvalho GA: Resection of large petroclival meningiomas by the simple retrosigmoid route. J Clin Neurosci 6:27-30, 1999

23. Savardekar AR, Goto T, Nagata T, Ishibashi K, Terakawa Y, Morisako H, et al: Staged 'intentional' bridging vein ligation: a safe strategy in gaining wide access to skull base tumors. Acta Neurochir (Wien) 156:671-679, 2014

24. Steiger HJ, Hänggi D, Stummer W, Winkler PA: Customtailored transdural anterior transpetrosal approach to ventral pons and retroclival regions. J Neurosurg 104:38-46, 2006

25. Suzuki Y, Matsumoto K: Variations of the superficial middle cerebral vein: classification using three-dimensional CT angiography. AJNR Am J Neuroradiol 21:932-938, 2000

26. Tomio R, Akiyama T, Ohira T, Horikoshi T, Yoshida K: Usefulness of facial nerve monitoring for confirmation of greater superficial petrosal nerve in anterior transpetrosal approach. Acta Neurochir (Wien) 156:1847-1852, 2014

27. Wolf BS, Huang YP, Newman CM: The superficial sylvian venous drainage system. Am J Roentgenol Radium Ther Nucl Med 89:398-410, 1963

28. Yaşargil MG, Damur M: Thrombosis of the cerebral veins and dural sinuses, in Newton TH, Potts DG (eds): Radiology of the Skull and Brain. Book 4: Specific Disease Processes. St Louis: Mosby, 1974, Vol 2, pp 2375-2400

29. Zhao JC, Liu JK: Transzygomatic extended middle fossa approach for upper petroclival skull base lesions. Neurosurg Focus 25(6):E5, 2008

\section{Disclosure}

The authors report no conflict of interest concerning the materials or methods used in this study or the findings specified in this paper.

\section{Author Contributions}

Conception and design: Shibao, Toda, Yoshida. Acquisition of data: Shibao. Analysis and interpretation of data: Shibao, Yoshida. Drafting the article: Shibao. Critically revising the article: Toda, Yoshida. Reviewed submitted version of manuscript: Toda, Fujiwara, Yoshida. Administrative/technical/material support: Yoshida. Study supervision: Yoshida. Illustrator: Orii.

\section{Supplemental Information}

Previous Presentation

The manuscript was accepted as a podium presentation at the

11th annual meeting of the European Skull Base Society on June 27th, 2014, in Paris, France.

\section{Correspondence}

Shunsuke Shibao, Department of Neurosurgery, Keio University School of Medicine, 35 Shinano-machi, Shinjuku-ku, Tokyo 1608582, Japan. email: pochisuke616@mac.com. 